\title{
Femtosecond Laser Amplification Based on Stimulated Raman Scattering in Optical Fibers
}

\author{
Hani Jassim Kbashi \\ Department of Physics, College of Science, University of Baghdad, Baghdad, Iraq \\ E-mail: hani_saka@yahoo.com \\ Kais A. al-Naimee \\ Department of Physics, College of Science, University of Baghdad, Iraq \\ Istituto Nazionale di Ottical Applicata, INOA, University of Florence, Italy \\ E-mail: kais.al-naimee@ino.it \\ Hussein Jawad \\ Institute of Laser for Postgraduate Studies, University of Baghdad, Iraq \\ E-mail: hussein59hs@yahoo.com \\ Roberto Benocci, V.Narayanan \& Dimitri Batani \\ Dipartimento di Fisica “G.Occhialini”, Università di Milano Bicocca, Milano, Italy \\ E-mail: batani@mib.infn.it
}

This work is supported by LANDAU NETWORK-CENTRO VOLTA with the Programme of the Italian Minister of University for the "Requalification of the Iraqi University System".

\begin{abstract}
The amplification of femtosecond laser has been experimentally investigated around $1.060 \mu \mathrm{m}$ wavelength through an optical fiber based on stimulated Raman scattering (SRS). A passively mode locked Nd:Glass laser (model Timebandwidth GLX 200) giving a pulse duration of about $200 \mathrm{fs}$ at $1.060 \mu \mathrm{m}$ wavelength and $120 \mathrm{~mW}$ average optical power with $100 \mathrm{MHz}$ repetition rate is used as seed signal. Whereas the high repetition rate $88 \mathrm{MHz}$ Nd:YLF laser operating at $1.053 \mu \mathrm{m}$ with pulse duration of 120 Ps is used as pump laser of the 15 and 25 $\mathrm{m}$ long single mode fiber. The broadening in the time of the pulse is done in the first few meters of the optical fiber and then amplified due to the energy transfer from pump laser source to the seed laser pulse. The results showed that the peak power conversion efficiency exceeds $45 \%$ and the on-off peak gain is about $2 \mathrm{~dB}$. The peak wavelength shift is about $0.3 \mathrm{~nm}$ when the optical fiber is forward pumping by Nd:YLF laser. The self phase modulation (SPM) leads to compress the laser spectral width from $6.96 \mathrm{~nm}$ to $5 \mathrm{~nm}$ with small effect on the maximum nonlinear phase shift.
\end{abstract}

Keywords: Femtosecond laser, Raman amplifier, Stimulated Raman scattering, Passively mode locked

\section{Introduction}

Femtosecond mode-locked lasers are widespread to various scientific and engineering fields such as ultrafast spectroscopy [Bellini et.al. 2004, Naumov et.al. 2001], metrology[Kinoshita et.al. 2001], superfine material processing[Von et.al. 2001], microscopy[Hong et.al 2009, Ozaki et.al 2009], high harmonic generation[Villoresi et.al 2001, Merdji et.al. 2000] and so on.

Raman amplification in optical fiber is based on Raman scattering. Raman scattering is divided into two categories called spontaneous Raman scattering and stimulated Raman scattering. Spontaneous Raman scattering is an interaction between light and a material that causes some of the incident light to be shifted to different frequencies spontaneously. Light down shifted in frequency from the incident frequency is called a Stokes shift and up-shifted frequency is called an anti-Stokes shift. The amount of the frequency shift depends on the 
vibration modes (optical phonons) of the material[Grote and Venhgaus 2001. SRS in optical fibers has been mainly used for broadband amplifiers, tunable fiber lasers or pulse compressors [Agrawal 1995]. All these applications dealt only with the downshifted generate from the frequency difference between the pump and the signal known Stokes shift $\omega_{R}=\omega_{p}-\omega_{s}$, where amplification of seed signal copropagating with a pump occurs when the signal wavelength falls in the Raman gain bandwidth [Agrawal 1995]. The bandwidth of fiber Raman amplifier is suitable for amplification of short optical pulses and offers the potential to generate gain at any arbitrary wavelength with an appropriate pump source. This has provided a very effective and successful way of providing gain at those wavelengths not directly available with other amplifier such as rare-earth doped fibers [Christophe et.al. 2003]. Optical amplification via SRS has advantages of self-phase matching between the pump and the signal, wider bandwidth and low noise, which leads to increase system capacity, distance [Kim et.al. 2002] and high speed response, compared with other nonlinear processes. Fiber Raman amplifier (FRA) may be used to overcome the fiber loss in soliton based communication system.

With the common assumption that the intensity of signal remains much smaller than the pump intensity, the Raman amplification gain can be expressed as.

$$
G_{A}=\exp \left[\frac{g_{R} P_{o} L_{e f f}}{K A_{\text {eff }}}\right]
$$

Where $\mathrm{P}_{\mathrm{o}}$ is the pump power, $\mathrm{A}_{\mathrm{eff}}$ is the effective core area of the fiber, $g_{R}$ is the Raman gain coefficient, $\mathrm{L}_{\mathrm{eff}}$ is the effective fiber length, and $\mathrm{K}$ is a numerical factor that account for polarization scrambling between the optical pump and signal. For complete polarization scrambling, as in conventional single mode fiber, $\mathrm{K}=2$.

Just to report a few important experimental and theoretical investigations on nonlinear SRS in optical fibers, we recall that: $23 \mathrm{~dB}$ signal gains were obtained from a $140 \mathrm{nsec}$ pump laser with $1.4 \mathrm{~W}$ peak pump power over $20 \mathrm{~km}$ long dispersion shifted fiber [Horiguchi et.al. 1992]; 30dB gain were obtained when a heavily germanium-doped optical fiber pumped by $350 \mathrm{~mW}$ [Dianov 1996]; the interaction between optical parabolic pulses in a Raman fiber amplifier were investigated and demonstrated experimentally in $5.3 \mathrm{~km}$ of non-zero dispersion shifted fiber presenting normal group velocity dispersion at the injected signal pulse wavelength of $1550 \mathrm{~nm}$ [Christophe and Guy 2005, Cyril and John 2003]. Electric Industries, Ltd. (SEI) has developed a discrete FRA utilizing a highly nonlinear fiber for 4-channel CWDM systems. By FRA technique, the transmission over single mode fiber with the length of $150 \mathrm{~km}$ was successfully achieved [Toshiyuki et.al. 2003].

In this work, we demonstrate and investigate experimentally the amplification of femtosecond laser pulses by using the nonlinear SRS in optical fibers with forward pumping.

\section{The Experimental Setup}

The Raman amplifier configuration with forward pumping is shown in Fig. 1.

In the present work, we used a passively mode locked Nd:Glass laser, model Timebandwidth GLX 200, equipped with semiconductor storable absorber mirrors [Keller et.al.2004, Klopp et.al. 2002, Druonet.al 2004, Zhang et.al. 2004] was installed in the laser laboratory of the University of Milano Bicocca [Canova et.al. 2005, Marangoni et.al 2007, Milani et.al. 2007]. The system is pumped by continuous wave laser diode. It generates $\mathrm{Sech}^{2}$-shape pulses of about $200 \mathrm{fs}$ and can be tuned from 1050 to $1064 \mathrm{~nm}$ at a fundamental repetition rate of $100 \mathrm{MHz}$ and with an average output power of $120 \mathrm{~mW}$.

The experiments reported here were performed with the laser operating at a wavelength centered at $1060 \mathrm{~nm}$ with 3-dB bandwidth of $6.96 \mathrm{~nm}$, and produced $\mathrm{Sech}^{2}$-shape pulse with duration of $200 \mathrm{fs}$. Also the system was adjusted to get an initially negative value of the chirp parameter in the laser pulse [Benocci et.al. 2009, Hani et.al. 2009]. The pump source was used in our work is Nd:YLF laser centered at a wavelength $1.053 \mu \mathrm{m}$, a pulse duration of $120 \mathrm{Ps}, 88 \mathrm{MHz}$ repetition rate with $2 \mathrm{~W}$ output power. This laser is also provided of the control system, which allows an automatic optimization of the pulse train stability in a active mode-locked Nd:YLF laser cavity[Canova et.al 2005, Marengoni et.al. 2006.

The pump and the seed lasers were multiplexed and launched into the optical fiber through an optical D-coupler and propagate along with the fiber. To maximize the pump coupling efficiency, the spot is focused on the fiber core. The minimum attainable spot size at the focus of the coupling lens is governed by diffraction $(10 \mu \mathrm{m})$. A beam collimator was inserted before the coupling lens. The F230FC Thorlabs fiber collimation package is designed to focus laser beam to propagation through the fiber. We measured the output spectra for two fiber lengths (15 and 25m) using Ocean Optics spectrometer. 


\section{Results and Discussion}

The spectrum of the Nd:YLF pump laser source at a wavelength $1.053 \mu \mathrm{m}$ and the spectrum of the Nd:Glass femtosecond laser before propagation in optical fiber is shown in Figure 2. The $3 \mathrm{~dB}$ bandwidth of the Nd:YLF laser is about $0.8 \mathrm{~nm}$, while the $3 \mathrm{~dB}$ bandwidth of the Nd:Glass femtosecond laser is about $6.96 \mathrm{~nm}$. The Stokes shift $\Omega_{R}$ between the signal and the pump, plays an important role in the SRS process. In the present work, the pump wavelength was selected $1.053 \mu \mathrm{m}$ to achieve the desired gain bandwidth with the help of the composite Raman gain spectrum $\Omega_{R}$ which is equal to $12 \mathrm{THz}$. The optical fibers utilized are 15 and $25 \mathrm{~m}$ single mode optical fiber. Figures 3 and 4 show the spectrum of Nd:YLF pump laser and Nd:Glass femtosecond laser amplification by SRS for 15 and $25 \mathrm{~m}$ fiber length respectively. The input pump power was used in our work is 2 $\mathrm{W}$, the Raman gain coefficient is $1 * 10^{-13} \mathrm{~m} / \mathrm{W}$ for $12 \mathrm{THz}$ Stokes shift ${ }^{[10]}$, and the fiber length are 15 and $25 \mathrm{~m}$. Figure 5 represent the effect pump of the Nd:Glass laser spectra. The observed gain can be attributed to SRS due to the energy transfer from the high power pump laser to the femtosecond seed laser. The peak wavelength shifted about $0.3 \mathrm{~nm}$, spectral width narrowing from $6.96 \mathrm{~nm}$ to $5 \mathrm{~nm}$ due to the SPM and small effect of the maximum nonlinear phase shift were observed for $200 \mathrm{fs}$ laser pulses.

Tacking the integral of the spectrum on/off output signals and dividing the resulting signal pump on power with the pump off power gives us the experimental measured pump on/off signal gain, which is about $1.8 \mathrm{~dB}$. While the Raman gain for the signal is evaluated using equation 1 which is equal to $2 \mathrm{~dB}$, by using the parameter values typical of standard single mode optical fiber, $A_{\text {eff }}=50 \mu \mathrm{m}$ and $\mathrm{K}=2$, Raman gain coefficient is $1 * 10^{-13} \mathrm{~m} / \mathrm{W}$, and the pump power is $2 \mathrm{~W}$. Raman gain of Nd:Glass femtosecond laser pulses depends on the optical fiber length. We conclude that the optical excitation of the Ti:Sapphire gain medium is a useful candidate for fs laser pulse amplification as well. The all fiber ultrashort laser pulse amplifier based SRS doesn't appear any effect on the pulse length. The femtosecond laser amplification using the power transfer between the shorter wavelength to longer wavelength in standard optical fiber is about $2 \mathrm{~dB}$ for $25 \mathrm{~m}$ fiber length. The SPM leads to compress the laser spectral width with small effect on the maximum nonlinear phase shift.

\section{Conclusion}

Raman gain of Nd:Glass femtosecond laser pulses depends on the optical fiber length. We conclude that the optical excitation of the Ti:Sapphire gain medium is a useful candidate for fsec laser pulse amplification as well. The all fiber ultrashort laser pulse amplifier based SRS doesn't appear any effect on the pulse length. The femtosecond laser amplification using the power transfer between the shorter wavelength to longer wavelength in standard optical fiber is about $2 \mathrm{~dB}$ for $25 \mathrm{~m}$ fiber length. The SPM leads to compress the laser spectral width with small effect on the maximum nonlinear phase shift.

\section{References}

Agrawal G.P. (1995). Nonlinear Fiber Optics. $\left(2^{\text {nd }}\right)$. Academic Press Inc., San Diego Chapter 8.

Bellini M., Stefano C., Chiara C., Roberto E. and Marzia M. (2004). High resolution spectroscopy in the XUV with pairs of mutually coherent and time-delayed laser harmonics. Laser and Particle Beams, 22, 199-200.

Benocci R., Batani D., Jawad H., Carpeggiani P., Uday K., Levchenko A., Venkatakrishanan N. (2009). An automated autocorrelator for the measurement of $\mathrm{HF}$ femtosecond pulses.

Canova F., Marangoni C., Librizzi M., G.Lucchini, Batani D., Kilpio A., Shashkov E., Stuchebrukhov I., Vovchenko V., Chernomyrdin V., Krasuyk I., Hall T., Bittanti S. (2005). Development of the 10 Terawatt “ATTILA" Nd laser system. Radiation Effects and Defects in Solids, 160, 10-12, 669 - 675.

Canova F., Marangoni C., Librizzi M., Lucchini G., Dimitri B., Kilpio A., Shashkov E., Stuchebrukhov I., Vovchenko V., Chernomyrdin V., Krasuyk I., Hall T. and Bittanti S. (2005). Development of the 10TW 'ATTILA Nd laser system. Radiation Effects \& Defects in Solids, 160, 10-12, 669-675.

Christophe F. and Guy M. (2005). Interaction between optical parabolic pulses in a Raman fiber amplifier. OPTICS EXPRESS, 13, 15, 5825-5830.

Christophe F., Guy M., Cyril B. and John M. (2003). Experimental generation of parabolic pulses via Raman amplification in optical fiber. OPTICS EXPRESS, 11, 13, 1545-1552.

Cyril B. and John M. (2003). Experimental generation of parabolic pulses via Raman amplification in optical fiber. OPTICS EXPRESS, 11, 13, 1547-1552.

Dianov E. (1996). Raman Fiber Amplifier for the Spectral Region Near 1300nm. Laser Physics, 6, 3. 
Druon F., Balembois F., and Georges P. (2004). Ultra short pulsed and highly efficient diode pumped Yb:SYS mode locked oscillators. Optics Express, 12, 20, 5005- 5012.

Grote N., and Venhgaus H. (2001). Springer. Fiber Optic Communication Devices. (chapter 2)

Hani J., Hussein J., Kais N., Roberto B., Paolo C., Perego C. and Dimitri B. (2009). Spectrial width variation of ultrashort laser pulses in Monomode optical fibers. Journal of Nonlinear Optical Physics and Materials, 18, 3, 541-552.

Hong, W., Y. He, T. Wen, H. Du, J. Teng, X. Qing, Z. Huang, W. Huang, H. Liu, X. Wang, X. Huang, Q. Zhu, Y. Ding and H. Peng. (2009). Spatial and temporal characteristics of X-ray emission from hot plasma driven by a relativistic femtosecond laser pulse. Laser and Particle Beams, 27, 01, 19-26.

Horiguchi T., Sato T., and Koyamada Y. (1992). Stimulated Raman Amplification of 1.6um-band Pulsed Light in Optical Fibers. IEEE Photonics Technology Letters, 4, 1.

Keller W., Kartner K. Kopf. F, Braun D., Jung B., Fluck D. Honninger R., Matuschek C., Aus N., and Au, J. (2004). Semiconductor storable absorber mirrors (SESAM's) for femtosecond to nanosecond pulse generation in solid-state lasers. IEEE Journal of selected topics in Quantum Electronics, 2, 3 435-453.

Kim P., Park J., Yoon H., Park J., and Park N. (2002). In situ design method for mutichannel gain of a distributed Raman amplifier with multiwave OTDR. IEEE Photonics Technology Letters, 14, 12.

Kinoshita K., Harano H., Yoshii K., Ohkubo T., Fukasawa A., NakamuraK. and Uesaka M. (2001) Time-resolved X-ray diffraction at NERL. Laser and Particle Beams, 19, 01, 125-131.

Klopp P., Petrov V., and Griebner U. (2002). Passively mode locked Yb:KYW laser pumped by a tapered diode laser. Optics Express, 10, 2, 108-113.

Marangoni C., Canova F., Librizzi M., Benocci R., Narayanan V., Gomareschi M., Lucchini G., Batani D., Kilpio A., Shashkov E., Stuchebrukhov I., Vovchenko V., Chernomyrdin V., Krasuyk I., Hall T., Bittanti S. (2007). Mode-locking optimization with a real time feedback system in Nd:YLF laser cavity. Review Scientific Instruments, 78, 013105.

Marengoni C., Canova F., Dimitri B., Benocci R, Librizzi M., Gomareschi M., Lucchini G., Kilpio A., Shashkov E., Stuchebrukhov I., Vovchenko V., Chernomyrdin V., Krasuyk I., Hall T., and Bittanti S. (2006). Mode-locking optimization with a real-time feedback system in a Nd:yttrium lithium fluoride laser cavity. Review of Science Instruments, 77, 1, 1-8.

Merdji H., Guizard S., Martin P., Petite G., Quere F., Carre B., Hergott J., Deroff L., Salieres P., Gobert O., Meynadier P. and Perdrix M. (2000). Ultrafast electron relaxation measurements on alpha $-\mathrm{SiO}_{2}$ using high-order harmonics generation. Laser and Particle Beams, 18, 03, 489-494.

Milani M, Ferraro L., Causa F., Batani D. (2007). Lasing properties and nonlinearities of dyes under high power pumping. Laser and Particle Beams, 25, 1-10.

Naumov A., Zheltikov A., Fedotov A.,. Sodorov D, Tarasevitch A., Zhouand D., Von Der L. (2001). Ionization and absorption effects in high-order harmonic generation in gas-filled hollow fibres. Laser and Particle Beams, 19, 01, 75-79.

Ozaki T., Kieffer J, Toth R., Fourmaux S. and Bandulet H. (2009). Experimental prospects at the Canadian advanced laser light source facility. Laser and Particle Beams, 24, 01, 101-106.

Toshiyuki M., Masato T., Junko K., Tetsufumi T., Toshiaki O., Motoki K. and Masayuki S. (2004). Development of Highly-Nonlinear-Fiber-Based Raman Amplifier for CWDM Transmission Systems. SEI Technical Review, 57, 1, 7-11.

Villoresi P., Barbiero P., Poleftto L., Nisoli M., G. Cerullo, E. Priori, Stagira S., Delisio C., Bruzzese R. and Altucci C. (2001). Study of few-optical-cycles generation of high-order harmonics. Laser and Particle Beams, 19, 01, 41-45.

Von L., Sokolowski K., Blome C., Dietrich C., Zhou P., Tarasevitch A., Cavalleri A., Siders C., Barty C., Squier J., Wilson K., Uschmann I. and Förster E. (2001). Generation and application of ultrashort X-ray pulses. Laser and Particle Beams, 19, 01, 15-22.

Zhang S., Wu, E. Haifeng P. and Heping Z. (2004). Passive mode locking in a diode-pumped Nd:GdVO4 laser with a semiconductor storable absorber mirror. IEEE Journal of Quantum Electronics, 40, 5, 505- 508. 


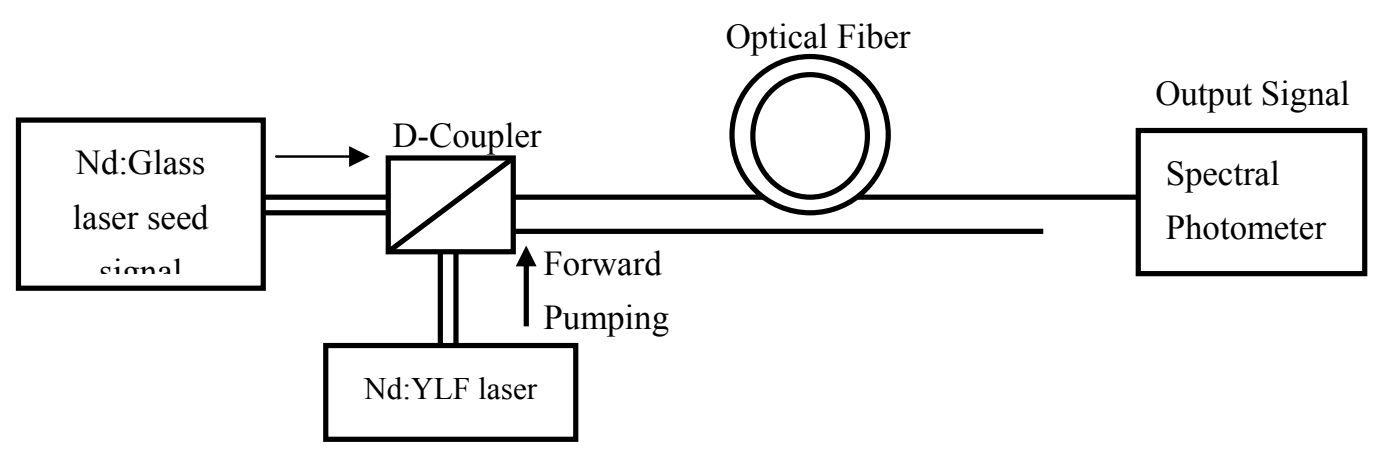

Figure 1. The experimental setup of the SRA with the forward pumping

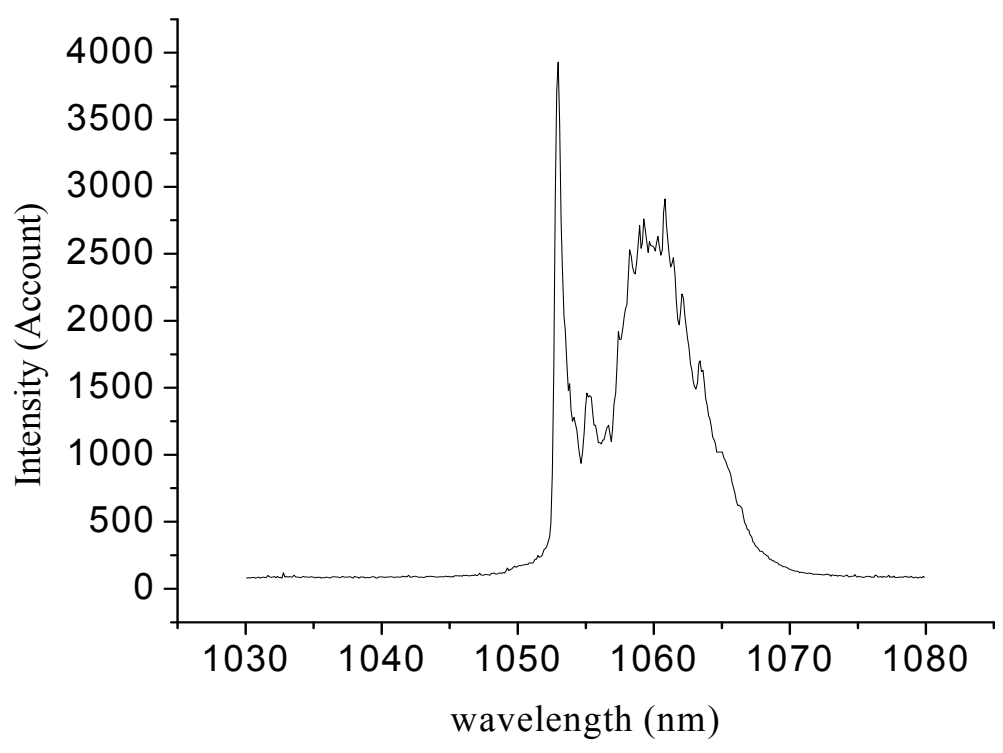

Figure 2. Experimental pulse spectra before amplification

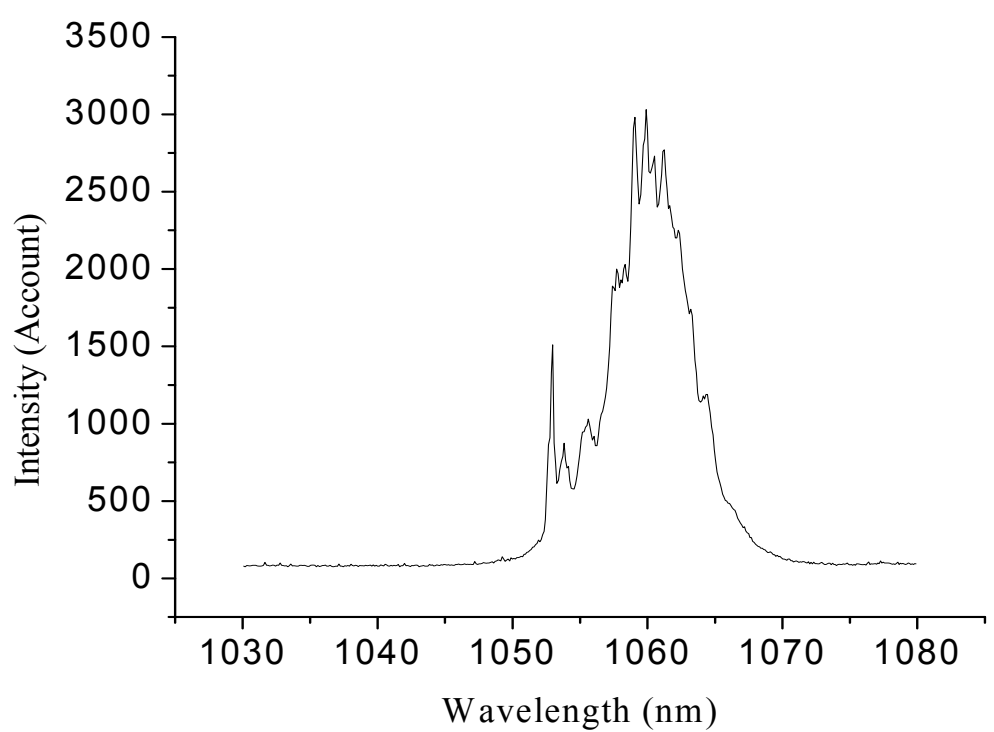

Figure 3. Experimental pulse spectra after $15 \mathrm{~m}$ fiber length amplification 


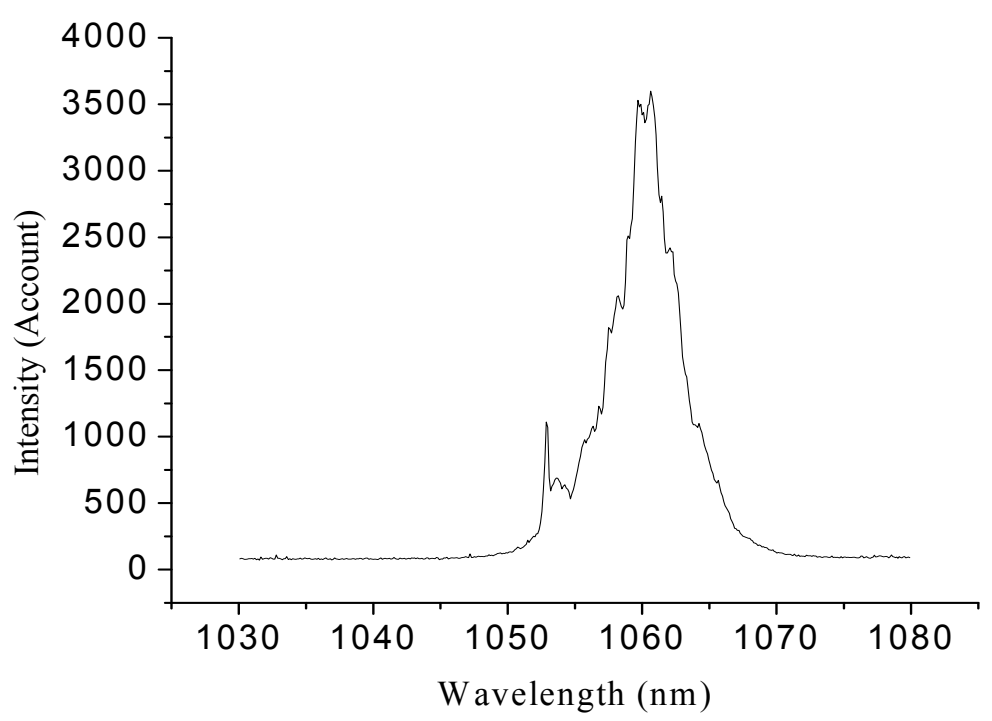

Figure 4. Experimental pulse spectra after $25 \mathrm{~m}$ fiber length amplification

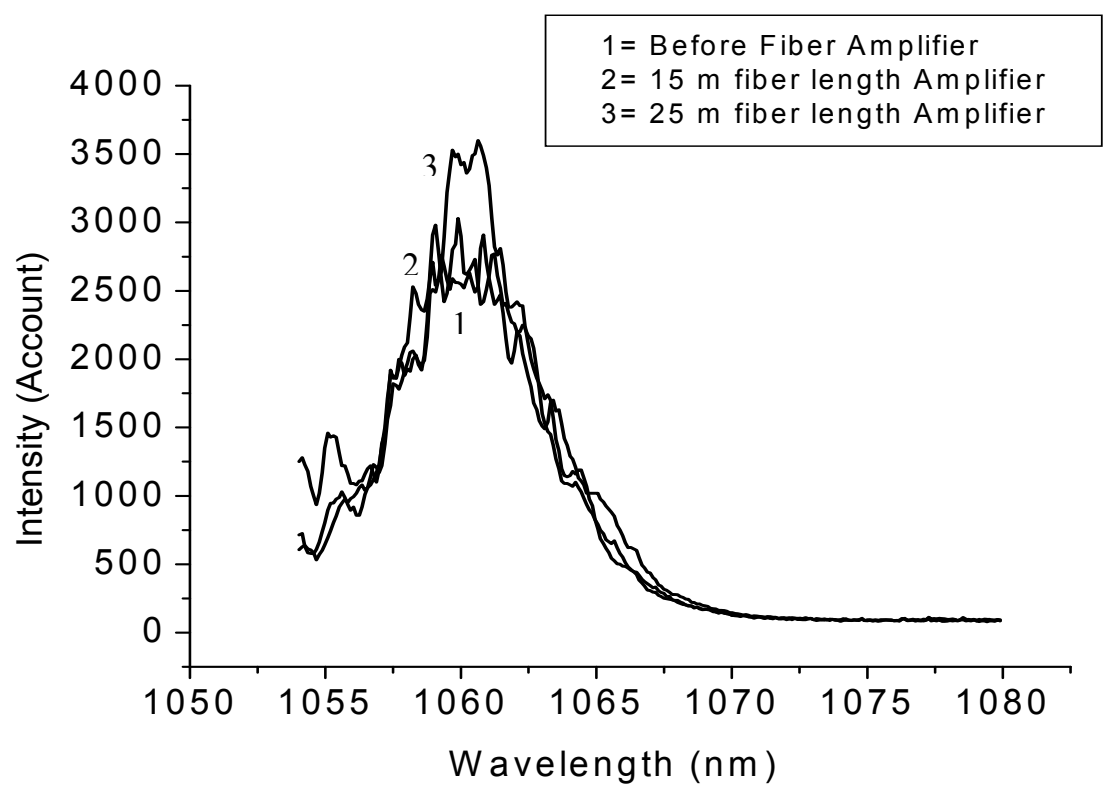

Figure 5. Experimental pulse spectra of ON/OFF amplification 